\title{
Manual para la realización de albarradas y fajinas para el control de la erosión en zonas forestales afectadas por el incendio de Hellín (Albacete) de 2012
}

\author{
Gómez-Sánchez E. ${ }^{* 1}$, Navarro T. ${ }^{2}$ \\ ${ }^{1}$ Servicio de Montes y Espacios Naturales Protegidos. Servicios Periféricos de la Consejería de Agricultura \\ en Albacete. Junta de Comunidades de Castilla la Mancha. C/ Mayor 4702071 Albacete (España). \\ ${ }^{2}$ Empresa Pública de Gestión Medioambiental de Castilla la Mancha. \\ Avda. España, 12. Sexta Planta. 02002 Albacete. \\ *e-mail: megomez@jccm.es
}

\section{Resumen}

En julio de 2012 se produjo en Hellín (Albacete) un incendio forestal que afectó a 5450.56 ha. En esta zona del sureste peninsular la recuperación de las cubiertas vegetales tras el fuego es muy lenta debido al escaso y torrencial régimen de precipitaciones, lo que aumenta las pérdidas de suelo e incrementa el riesgo de desertificación. Dentro del Plan de restauración de la zona afectada elaborado por el Servicio Forestal de la JCCM (Junta de Comunidades de Castilla la Mancha) se planteó, entre otras actuaciones urgentes, la realización de albarradas y fajinas en las zonas con mayor riesgo de erosión utilizando el material vegetal quemado. La limitación en recursos económicos, la escasez en algunas zonas del material vegetal para la ejecución de las estructuras, así como la necesidad de actuar en la mayor superficie incendiada posible antes del primer episodio de lluvias torrenciales, llevó a la elaboración de un "Manual para la ejecución de albarradas y fajinas para el control de la erosión”, a fin de optimizar rendimientos y garantizar la efectividad de los trabajos. Dicho manual contempla una clasificación previa del área de trabajo en función de su sensibilidad al riesgo de erosión, criterios de actuación en cada zona y finalmente los criterios constructivos de las estructuras. Una vez ejecutados estos trabajos se presentan algunos resultados recopilados.

Palabras clave: Albarradas, desertificación, estructuras vegetales, fajinas, gestión montes quemados. 


\section{Introducción}

La degradación del suelo y el riesgo de erosión por la pérdida de la cubierta vegetal suelen ser los procesos más críticos después de un incendio (Vallejo, 2009), máxime en las zonas semiáridas de la Cuenca Mediterránea donde la cubierta vegetal, sometida a fuerte estrés hídrico, presenta una cobertura muy pobre los meses posteriores al incendio (Pausas y Vallejo, 1999) coincidiendo con el inicio del periodo otoñal de lluvias torrenciales. Ello hace incrementar el riesgo de desertificación (Bautista, 1999).

Por ello, independientemente de la mayor o menor resiliencia del sistema tras el fuego - esto es, la capacidad de un ecosistema para retornar a las condiciones previas al incendio que entre otros factores dependerá de la severidad, recurrencia, estrategias adaptativas de la vegetación, etc.-, la gestión de las zonas forestales quemadas pasa por la rápida planificación y ejecución de actuaciones efectivas encaminadas a prevenir la erosión hídrica (Alloza, 2013), máxime en climas áridos. Con este fin, suele ser habitual la realización de sencillas estructuras vegetales manuales (albarradas y fajinas) en los meses inmediatamente posteriores al fuego con el fin de retener el suelo, reduciendo el volumen de sedimentos arrastrados, y disminuir la energía de escorrentía (Vallejo et al., 2007; Chavarría y López de Diego, 2008; Jordán et al.,

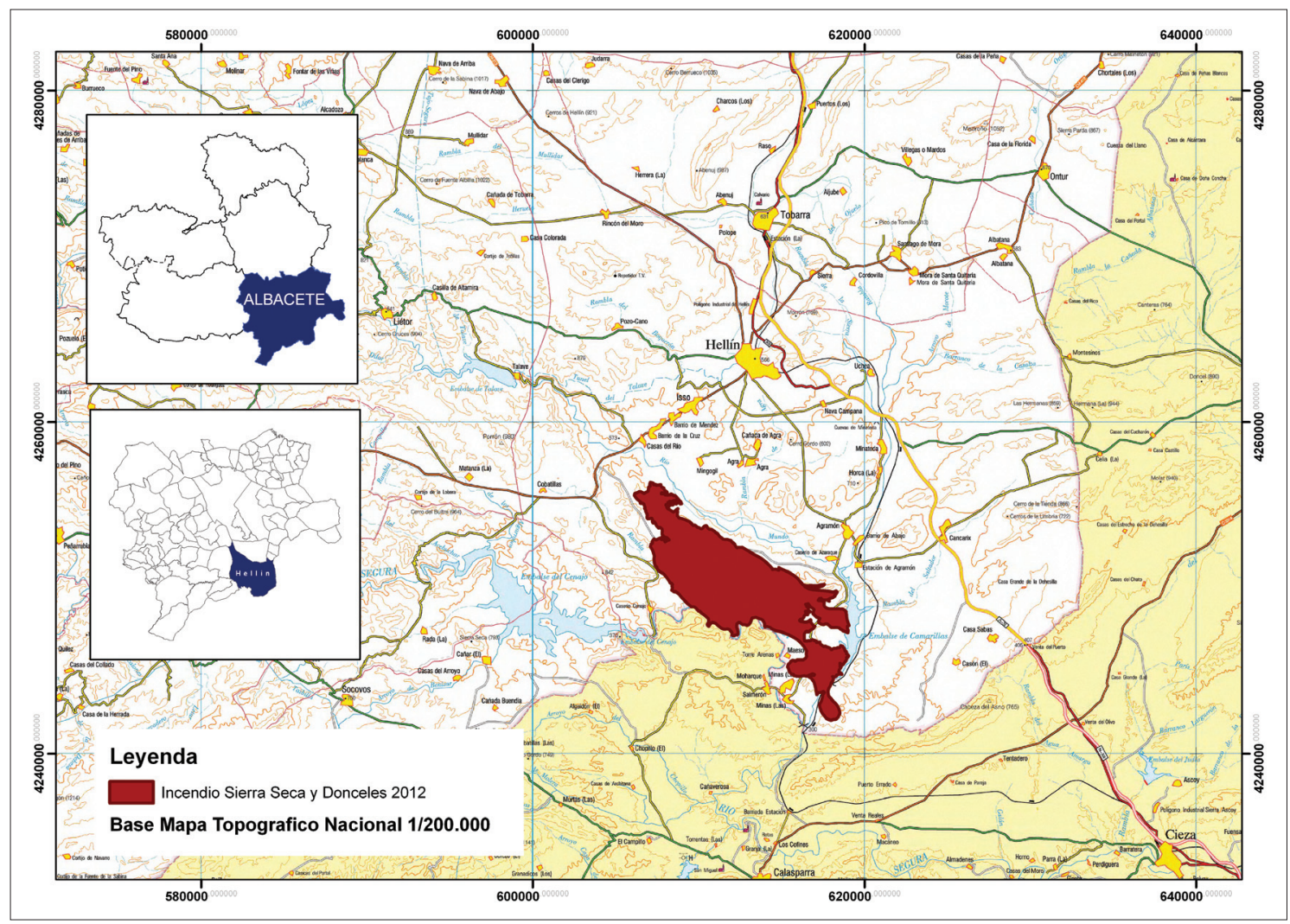

Figura 1. Mapa de localización de la superficie afectada por el incendio de Sierra Seca y Donceles de 1 de julio de 2012. 
2013); recomendándose la prescripción de sus características técnicas acorde a la erosionabilidad del suelo (Hernández y Romero, 2008).

\section{Descripción de la zona del incendio}

La zona incendiada (Fig. 1) pertenece a la cuenca hidrográfica del Segura estando encuadrada geológicamente en la zona Prebética. Los materiales que conforman esta zona son en su mayoría calizas más o menos dolomitizadas, margas y arcillas. La altitud de la zona incendiada, oscila entre $314 \mathrm{~m}$ y los $808 \mathrm{~m}$. Los suelos son calizos en su mayoría, con zonas yesíferas.

Según clasificación climática de Rivas Martínez nos encontramos en el piso mesomediterráneo de ombroclima seco a semiárido; con una temperatura media de $17^{\circ} \mathrm{C}$ y precipitación media de $350 \mathrm{~mm}$. Biogeográficamente la zona pertenece a la Provincia Murciano-Almeriense, distrito murciano-septentrional del subsector murciano. La zona quemada estaba ocupada por pinares de carrasco (Pinus halepensis) de origen natural en las áreas más húmedas e inaccesibles; pinares de repoblación realizadas hace 25-30 años por la Administración en zonas de escaso suelo y fuertes pendientes, con escaso desarrollo; y matorrales tipo espartizales, romerales y tomillares térmicos, a veces como vegetación principal y otras como acompañante del pino carrasco.

\section{Manual para la ejecución de albarradas y fajinas para el control de la erosión}

Dentro del Plan de restauración de la zona afectada por el incendio de Hellín de 1 de julio de 2012, elaborado por el Servicio Forestal, se incluyeron entre las acciones urgentes para el control de la erosión la construcción de albarradas y fajinas con el material quemado. La necesidad de mejorar el rendimiento de ejecución y la eficacia de la actuación condujo a la redacción de un Manual cuyo contenido se desarrolla a continuación.

\subsection{Zonificación previa de la zona quemada}

Previo a la ejecución de las estructuras se elaboró la cartografía de vegetación preincendio y de riesgo de erosión hídrica, esta última basada en la Ecuación Universal de Pérdidas de Suelo, que permitiera seleccionar las áreas de actuación (Fig. 2). Solapando las zonas con mayor riesgo de erosión y las zonas ocupadas por pinares más o menos densos (donde encontramos el material vegetal disponible para la construcción de las estructuras) se identificaron las superficies para la ejecución de albarradas y fajinas.

\subsection{Tipología de estructuras: definición y objetivos}




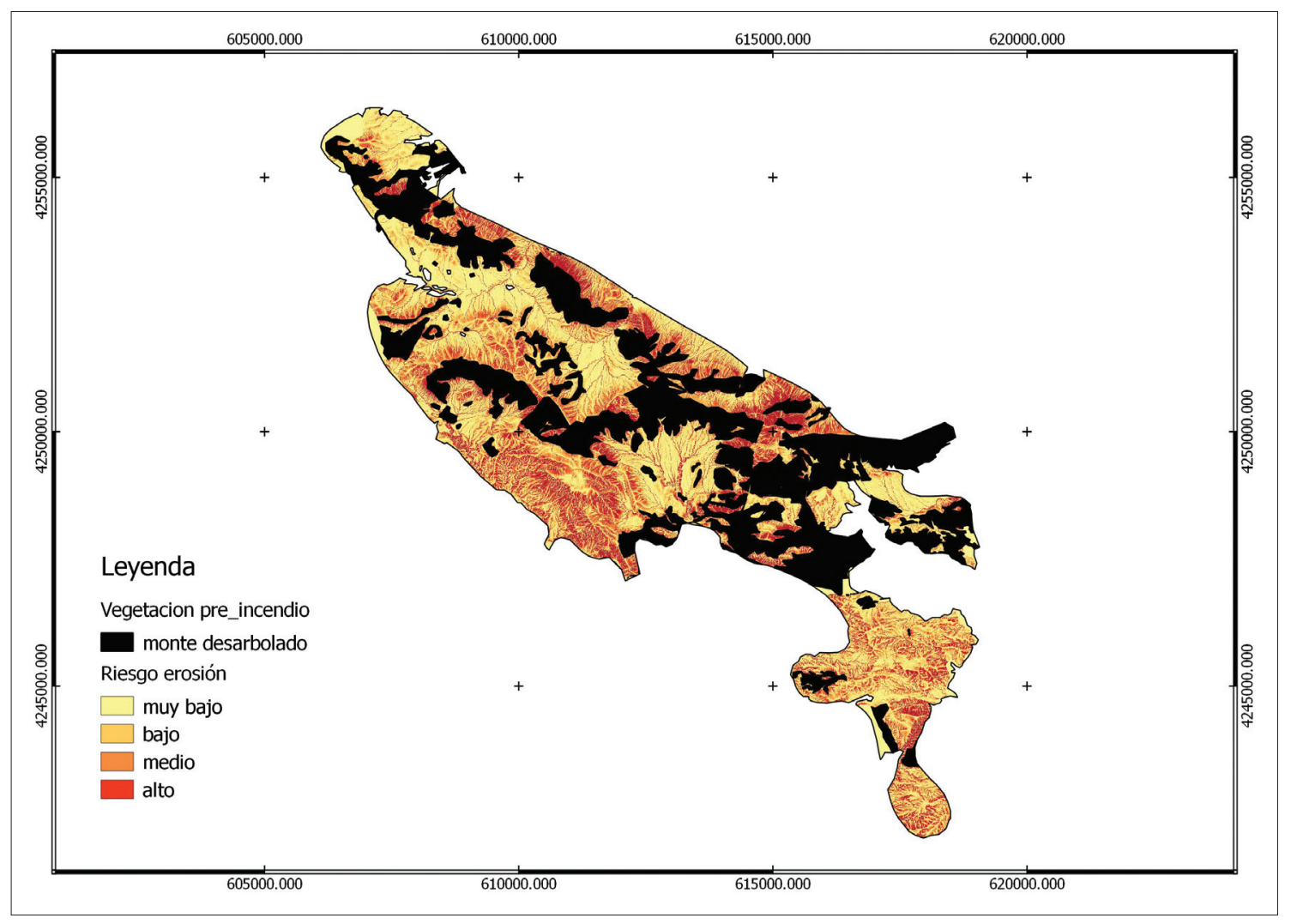

Figura 2. Mapa de riesgo de erosión hídrica de las zonas arboladas en el área afectada por el incendio de Sierra Seca y Donceles de 1 de julio de 2012.

Albarradas: Estructuras realizadas en los barrancos, canales de desagüe o cárcavas posicionadas perpendicularmente al flujo del agua con los objetivos de:

- Disminuir la velocidad de circulación del agua de escorrentía evitando el crecimiento de la cárcava (tanto lateral en talud y lecho como hacia la parte alta o inicio de la cárcava responsable de la erosión remontante).

- Actuar como barrera-filtro que retenga el material arrastrado.

- Facilitar la regeneración natural aguas arriba de la estructura al favorecer la acumulación de sustrato favorable.

Fajinas: Estructuras realizadas en ladera, construidas siguiendo curvas de nivel, diseñadas para disminuir la longitud efectiva de la pendiente con los objetivos de:

- Frenar el paso del agua disipando su energía cinética, y reduciendo con ello su velocidad a un nivel no erosivo.

- Actuar sobre el origen de las cárcavas, evitando su crecimiento.

- Proteger caminos frente arrastres de ladera.

- Favorecer acumulación de materiales, creando un microclima favorable para la colonización y desarrollo de la vegetación.

En las zonas que por razón de seguridad del personal (pendiente, pedregosidad,...), no se puedan realizar estas estructuras, se intentará apear los pies y se dejarán en el terreno preferentemente en dirección perpendicular a la escorrentía. Si exis- 
te riesgo de deslizamientos que pudieran ocasionar daños, se fijará el tronco al suelo con estacas y/o mediante encamado del tronco.

En el caso de exceso de material, éste se alineará en el monte formando cordones para facilitar posteriores trabajos de gestión o simplemente se esparcirá por el mismo; evitando en todo caso su depósito en cárcavas y ramblas.

\subsection{Materiales para la ejecución}

Las albarradas y fajinas se construirán empleando fustes y ramas de los pies quemados una vez apeados y procesados para su fácil manejo. Para ello se cortarán todos los pies quemados o parcialmente quemados, que tengan más de dos tercios de su altura total quemada o más de la mitad de la copa quemada, dejando un mínimo de 10 árboles ha-1 que actúen temporalmente como árboles percha o posaderos de avifauna y favorezcan la entomofauna saprofílica fomentando el papel funcional de la madera quemada; éstos se seleccionarán de entre los completamente quemados de tamaño medio y sin acículas situados en zonas inaccesibles o de difícil extracción.

\subsection{Criterios constructivos}

\subsubsection{Albarradas}

Las albarradas tendrán una altura media aproximada de $60 \mathrm{~cm}$. (no superando $10 \mathrm{~s}$ $100 \mathrm{~cm}$ ), longitud variable en función de la anchura de la cárcava y profundidad según grosor del fuste utilizado (Fig. 3).

Localización: El progreso de ejecución seguirá "criterio de cuenca" con el objetivo de contrarrestar la pérdida de suelo, de modo que se comenzarán a construir de arriba a abajo - en el sentido de circulación del agua- hasta que la cárcava tenga como máximo 1 metro de profundidad, altura máxima para la cual se consideran efectivas este tipo de construcciones.

En la colocación definitiva de la albarrada se buscará el sitio que mejor favorezca la retención de sedimentos, localizado en el punto en que la pendiente del lecho sea menor (Fig. 4).

Espaciamiento: La separación entre albarradas dependerá de la altura de la albarrada y la pendiente del lecho de la cárcava (Tab. 1).

Conviene no olvidar que son distancias ideales y que se pueden variar si eso permite un diseño más eficaz, como es el caso de ajustar su posición a una sección más estrecha donde su construcción conlleve menos dificultad y/o donde se carece de material suficiente y/o el caso de pendientes muy elevadas donde el espaciamiento se reduce considerablemente.

Ejecución. El proceso de construcción será el siguiente:

- Anclar estacas verticales clavadas (diámetros de más de $10 \mathrm{~cm}$, óptimo $15 \mathrm{~cm}$ y longitud suficiente según altura de la albarrada), aprovechando tocones cuando sea posible. La instalación comienza por el punto más bajo de la zona a reparar, hincando las estacas de madera verticalmente a una profundidad de aprox. $60 \mathrm{~cm}$ y con una distancia de entre 30-50 centímetros. 


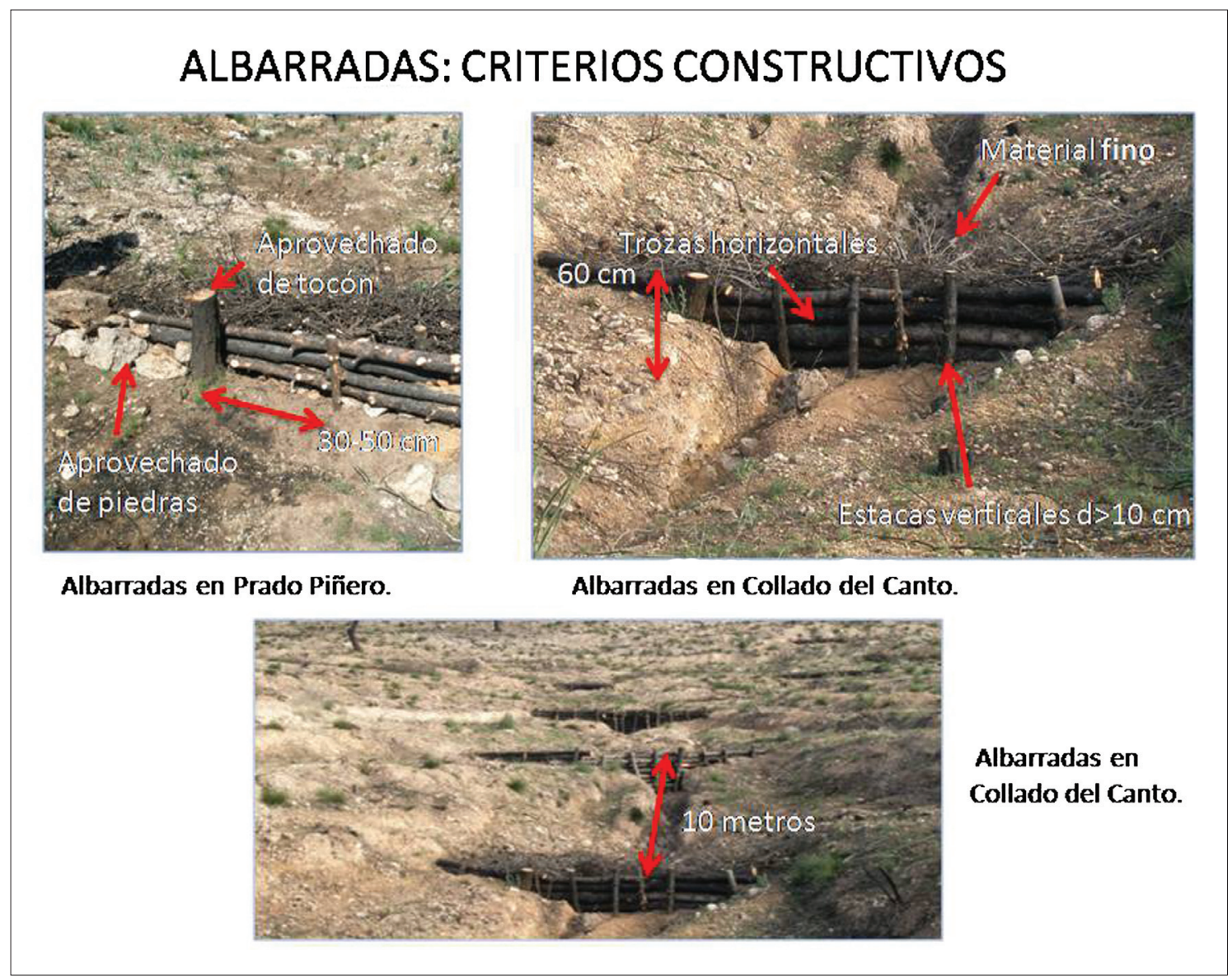

Figura 3. Albarradas. Criterios constructivos (d=diámetro estacas).

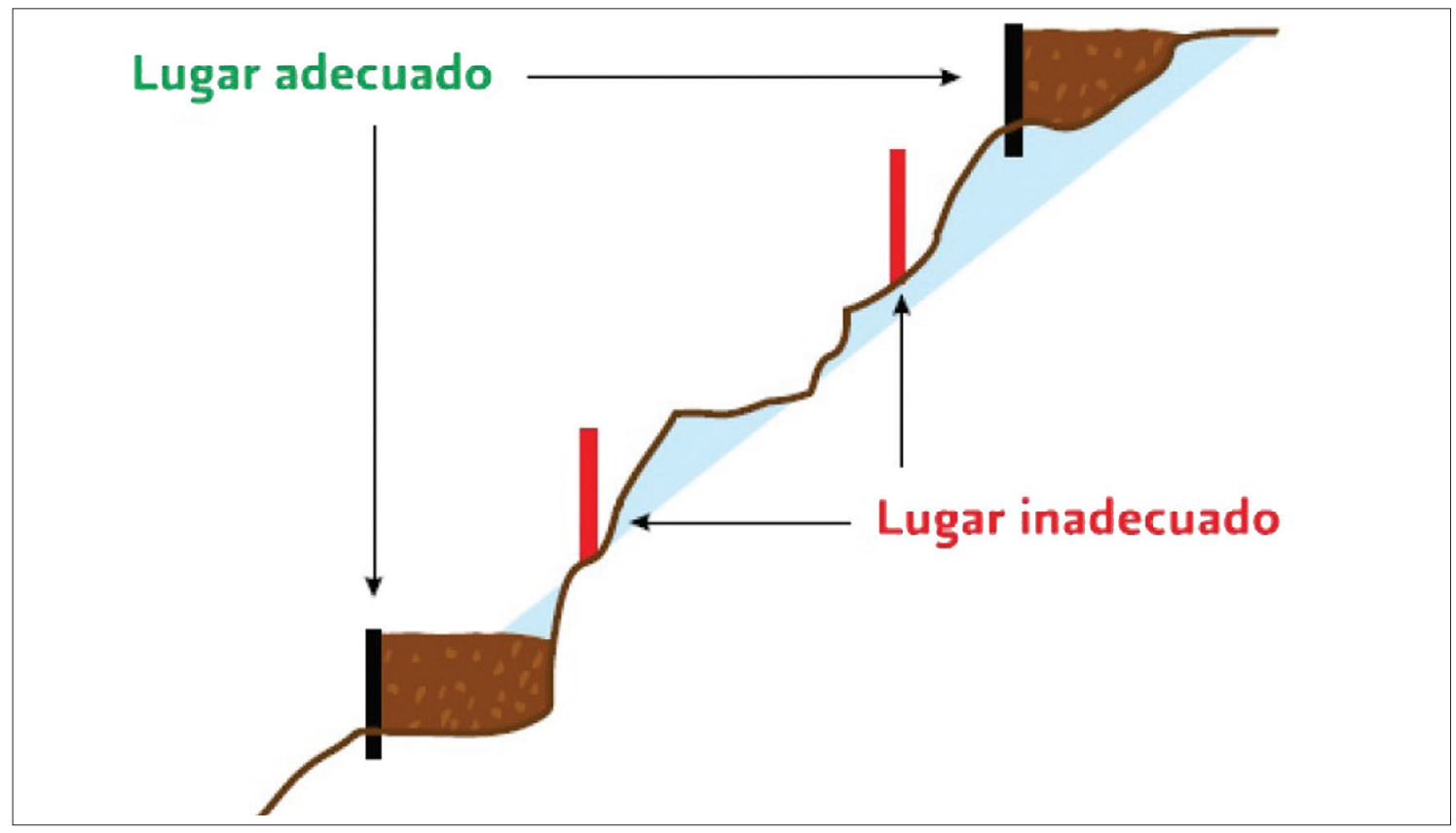

Figura 4. Localización de albarrada más eficiente según pendiente longitudinal de cárcava. 
Tabla 1. Separación de albarradas según altura de las mismas y pendiente de la cárcava.

\begin{tabular}{c|c|c|c|c|c}
\hline $\begin{array}{c}\text { Pendiente (\%) } \\
\text { Altura (cm) }\end{array}$ & 10 & 16 & 20 & 30 & 40 \\
30 & 10 & 6 & 5 & 2 & 2 \\
\hline 40 & 14 & 8 & 6 & 3 & 3 \\
\hline 60 & 20 & 13 & 10 & 4 & 4 \\
\hline 80 & 27 & 17 & 13 & 5 & 4 \\
\hline 100 & 34 & 20 & 17 & 7 & 5 \\
\hline
\end{tabular}

- Garantizar la retención de materiales en la estructura sellándola al suelo. Para ello recoger piedras próximas y empotrarlas en el lecho y talud y/o encamar los fustes de mayor diámetro dispuestos en horizontal en los laterales y fondo de la vaguada realizando si es necesaria una pequeña zanja abierta al efecto.

- Colocar troncos y ramas entrelazadas. Si se cree necesario se procederá al atado de troncos a los postes mediante cuerdas.

- Rellenar con material fino aguas arriba (evitar exceso de material).

- Si se considerara necesario para la estructura realizada y existen materiales en el terreno para ello, colocar troncos, ramas o rocas a modo de encachado aguas abajo de la estructura con el fin de disipar la energía y evitar socavamientos en los puntos de derrame. Se debe cubrir como mínimo la anchura del canal y $1 \mathrm{~m}$. de longitud desde el punto de derrame.

- Se cortarán los pies necesarios para construir las albarradas tal y como se especifica en el Apartado 3.3.

- No se abandonarán residuos/restos en las cárcavas de manera descontrolada. En el caso de exceso de restos se acordonarán o distribuirán de manera dispersa en la ladera.

\subsubsection{Fajinas}

Se dispondrán constituyendo estructuras perpendiculares a la escorrentía, en curvas de nivel sujetas por estacas o estaquillas de contorno o de contención (Fig. 5).

La altura óptima de la fajina será de $25 \mathrm{~cm}$. En el caso de fajinas por encima de carreteras o pistas forestales, su función será también la de servir de freno al material rodante, por lo que deberán ser más altas (en torno a $40 \mathrm{~cm}$ ).

La longitud de cada fajina dependerá de la topografía no superando valores de 15 $\mathrm{m}$, puesto que se complica la alineación plena con la curva de nivel.

La profundidad dependerá del material disponible y del diámetro de árboles cortados.

Localización: Se localizarán con carácter general en laderas con una pendiente mayor del $20 \%$ y en taludes de barrancos, independientemente de la pendiente de la ladera, evitando escurrimientos que dan lugar al crecimiento en anchura de los mismos. No se ejecutarán en: 


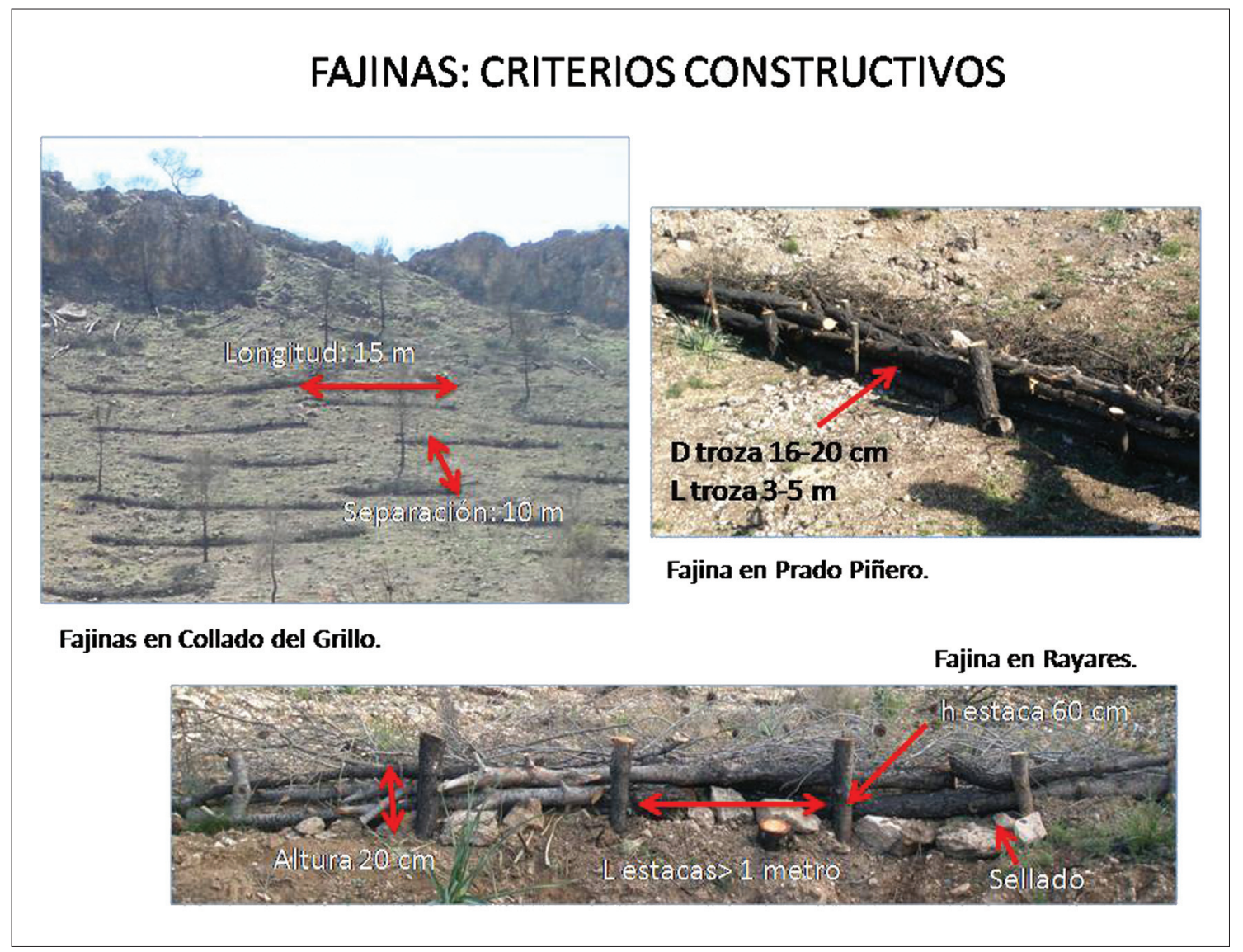

Figura 5. Fajinas. Criterios constructivos ( $\mathrm{D}=$ diámetro; $\mathrm{L}=$ longitud; $\mathrm{H}=$ Altura).

- Rodales con árboles soflamados o vivos, en definitiva áreas poco afectadas por el fuego.

- Laderas con pendientes superiores al 60\%, atendiendo a la seguridad del personal.

- Superficies rocosas o pedregosas con una cobertura de suelos mayor del $60 \%$. La baja erosión del suelo, en estos casos, no precisa la construcción de fajinas.

Al igual que en el caso de las albarradas, para las fajinas se aconseja progresar utilizando el "criterio de cuenca", ejecutando el trabajo en la ladera desde arriba hacia abajo. De este modo es más fácil identificar el flujo del agua ladera abajo y con ello determinar mejor la localización de las fajinas. Además, de esta forma se atiende a la mayor seguridad de los operarios.

Espaciamiento: La densidad de fajinas recomendada es de 500 metros lineales por hectárea. El criterio general, para su ordenamiento espacial, será su disposición a lo largo de curvas de nivel. Esto se conseguirá con mayor facilidad en terrenos con topografía homogénea, siendo de mayor complejidad con topografía heterogénea, donde la adaptación de la longitud de la fajina al terreno, facilitará su construcción 
más eficiente. La distancia media de separación entre fajinas será de $10 \mathrm{~m}$, aumentando si la línea de máxima pendiente del terreno disminuye y viceversa. La longitud continua de las construcciones no excederá de $15 \mathrm{~m}$, ante la dificultad para mantener el nivel. Las interrupciones de fajinas en diferente cota se realizarán contrapeadas (al tresbolillo), para salvar la posible erosión de esas separaciones.

Ejecución. El proceso de construcción será el siguiente:

- Colocar estacas o estaquillas de contorno. La sujeción de la estructura se realizará con carácter general mediante estacas o estaquillas de contención. La longitud de las estacas o estaquillas, no será inferior a los $60 \mathrm{~cm}$, y se situarán a una distancia no inferior al metro. Se debe poner especial atención en que queden bien ancladas. En muchos casos, por la disposición de los pies quemados, se podrá usar el tocón del propio pie cortado a modo de estaca de contención de la fajina.

- Colocación de la vegetación. Se dispondrán tanto ramas como trozas de fustes entrelazados formando un cordón continuo. Las trozas deben colocarse bien niveladas, perpendiculares a las líneas de máxima pendiente. En caso necesario, la troza será encamada realizando una pequeña trinchera, con relleno ladera arriba para pegarla y sellarla al suelo a fin de evitar que el agua fluya por debajo.

- El diámetro adecuado de las trozas es de 16 a $20 \mathrm{~cm}$, pudiendo ser mayores atendiendo a disponibilidad. En zonas donde los árboles sean menores pueden hacerse grupos de 3 a 6 trozas. La longitud de las trozas debe comprenderse entre los 3 y 5 metros, pues si fuesen de mayor longitud serían difíciles de manejar, siendo complicado su encamado en el suelo.

- El arbolado de gran tamaño caído o con riesgo de caída al suelo será asegurado contra tocones, ayudado con sus propias ramas u otros elementos naturales; o en caso necesario mediante estacas clavadas en el terreno para evitar que ruede ladera abajo. Asimismo, si es posible su uso para el control hidrológico, deberá alinearse con la curva de nivel comprobando que está perfectamente en contacto con el suelo, sin huecos entre medias, para conseguir un control adecuado de la escorrentía.

- En los casos en los que exista material en exceso se podrá realizar un cordón entre fajinas, pudiéndose ampliar la distancia inicial establecida para las fajinas.

- En los extremos de laderas partidas por cárcavas de cierta entidad se pondrá especial énfasis en ajustar las fajinas a la topografía, curvando la fajina según el talud y reduciendo las distancias entre fajinas.

\section{Resultados}

Para la ejecución de los trabajos se organizaron hasta 7 cuadrillas, distribuidas por las diferentes zonas de trabajo de manera simultánea para minimizar los tiempos de ejecución. Los trabajos a realizar por las mismas han consistido en el señalamiento de árbo- 
les "percha", apeo y procesado del arbolado quemado, localización óptima de las estructuras (albarradas y/o fajinas), construcción de estructuras con el material procesado, ajuste de la estructura al terreno y acordonado o esparcido del material sobrante.

Los resultados de la medición de los trabajos de restauración hidrológica ejecutados por medio de albarradas y fajinas se muestran en la Tab. 2, indicando por zonas y cuadrillas la superficie ejecutada y los jornales para ello, así como el número de estructuras realizadas y su densidad por unidad de superficie.

Tabla 2. Resultado de las mediciones de los trabajos ejecutados. Por cuadrilla y zona de trabajo se indican JOR EFECT $=$ Jornales efectivos (7 horas), SUP=superficie trabajada (ha), ALBARR=albarradas, FAJINA=fajinas de $15 \mathrm{~m}$, ESTRUCT=suma albarradas y fajinas expresadas en número total y por unidad de superficie $\left(\mathrm{ha}^{-1}\right)$.

\begin{tabular}{|c|c|c|c|c|c|c|c|c|c|}
\hline CUADRILLA & ZONA & JOR EFECT & SUP & ALBARR & FAJINA & ESTRUCT & ALBARR $^{\text {ha-1 }}$ & FAJINA $^{\text {ha-1 }}$ & ESTRUCT $^{\mathrm{ha}-1}$ \\
\hline Cuadrilla 1 & A & 165 & 10.06 & 43 & 20 & 63 & 4.27 & 1.99 & 6.3 \\
\hline Cuadrilla 2 & B & 96 & 3.38 & 5 & 70 & 75 & 1.48 & 20.71 & 22.2 \\
\hline Cuadrilla 3 & $\mathrm{~B}$ & 116 & 2.99 & 28 & 110 & 138 & 9.36 & 36.79 & 46.2 \\
\hline Cuadrilla 4 & $\mathrm{C}$ & 103 & 18.52 & 60 & 0 & 60 & 3.24 & 0.00 & 3.2 \\
\hline Cuadrilla 5 & $\mathrm{C}$ & 125 & 33.14 & 160 & 0 & 160 & 4.83 & 0.00 & 4.8 \\
\hline Cuadrilla 4 & $\mathrm{C}$ & 25 & 2.60 & 4 & 7 & 11 & 1.54 & 2.69 & 4.2 \\
\hline Cuadrilla 5 & $\mathrm{C}$ & 15 & 0.39 & 2 & 5 & 7 & 5.13 & 12.82 & 17.9 \\
\hline Cuadrilla 6 & $\mathrm{D}$ & 154 & 14.36 & 150 & 64 & 214 & 10.45 & 4.46 & 14.9 \\
\hline Cuadrilla 7 & $\mathrm{E}$ & 182 & 10.29 & 120 & 26 & 146 & 11.66 & 2.53 & 14.2 \\
\hline Cuadrilla 1 & A & 104 & 12.55 & 35 & 68 & 103 & 2.79 & 5.42 & 8.2 \\
\hline Cuadrilla 2 & B & 59 & 2.11 & 15 & 75 & 90 & 7.11 & 35.55 & 42.7 \\
\hline Cuadrilla 3 & B & 38 & 2.34 & 0 & 30 & 30 & 0.00 & 12.82 & 12.8 \\
\hline Cuadrilla 3 & $\mathrm{~F}$ & 15 & 2.21 & 9 & 1 & 10 & 4.07 & 0.45 & 4.5 \\
\hline Cuadrilla 4 & $\mathrm{C}$ & 54 & 14.36 & 91 & 0 & 91 & 6.34 & 0.00 & 6.3 \\
\hline Cuadrilla 5 & $\mathrm{C}$ & 92 & 20.71 & 100 & 0 & 100 & 4.83 & 0.00 & 4.8 \\
\hline Cuadrilla 6 & $\mathrm{D}$ & 58 & 11.29 & 55 & 0 & 55 & 4.87 & 0.00 & 4.9 \\
\hline Cuadrilla 7 & $\mathrm{D}$ & 115 & 26.76 & 230 & 0 & 230 & 8.59 & 0.00 & 8.6 \\
\hline Cuadrilla 1 & A & 102 & 18.21 & 59 & 56 & 115 & 3.24 & 3.08 & 6.3 \\
\hline Cuadrilla 2 & $\mathrm{~B}$ & 22 & 1.01 & 14 & 2 & 16 & 13.86 & 1.98 & 15.8 \\
\hline Cuadrilla 6 & G & 77 & 10.57 & 85 & 0 & 85 & 8.04 & 0.00 & 8.0 \\
\hline Cuadrilla 5 & $\mathrm{C}$ & 239 & 53.01 & 160 & 0 & 160 & 3.02 & 0.00 & 3.0 \\
\hline Cuadrilla 3 & $\mathrm{~F}$ & 164 & 23.99 & 75 & 0 & 75 & 3.13 & 0.00 & 3.1 \\
\hline Cuadrilla 7 & $\mathrm{D}$ & 294 & 50.79 & 420 & 0 & 420 & 8.27 & 0.00 & 8.3 \\
\hline Cuadrilla 1 & $\mathrm{H}$ & 146 & 21.04 & 88 & 107 & 195 & 4.18 & 5.09 & 9.3 \\
\hline Cuadrilla 6 & $\mathrm{G}$ & 162 & 31.64 & 180 & 0 & 180 & 5.69 & 0.00 & 5.7 \\
\hline Cuadrilla 1 & $\mathrm{H}$ & 54 & 13.05 & 25 & 6 & 31 & 1.92 & 0.46 & 2.4 \\
\hline Cuadrilla 6 & I & 114 & 25.62 & 140 & 0 & 140 & 5.46 & 0.00 & 5.5 \\
\hline Cuadrilla 5 & $\mathrm{C}$ & 60 & 8.39 & 10 & 10 & 20 & 1.19 & 1.19 & 2.4 \\
\hline Cuadrilla 4 & I & 90 & 12.61 & 50 & 0 & 50 & 3.97 & 0.00 & 4.0 \\
\hline TOTAL & 3040 & 458 & 2413 & 657 & 3070 & & & & \\
\hline
\end{tabular}


Se han construido un total de 3070 estructuras (2413 albarradas y 657 fajinas) en una superficie de 458 ha, con la participación de 3040 jornales. El promedio de estructuras para el total de la superficie ejecutada es de 10.36 estructuras ha-1.

Los rendimientos de las diferentes cuadrillas se representan en la Fig. 6. Cada cuadrilla tiene diferentes tiempos de desplazamiento al tajo asignado, así como una zona de trabajo distinta en términos de dificultad (topografía) e intensidad (número de estructuras a ejecutar); por ello hablamos de jornales efectivos por hectárea (Jor efect $\mathrm{ha}^{-1}$ ) obtenidos al descontar los tiempos de desplazamiento, que se comparan en función de la densidad de trabajo de cada zona (estructuras ejecutadas por superficie). La diferencia de trabajo entre la construcción de una albarrada y una fajina de $15 \mathrm{~m}$ se entiende que no es importante, por lo que se han englobado todas bajo el tipo "estructuras".

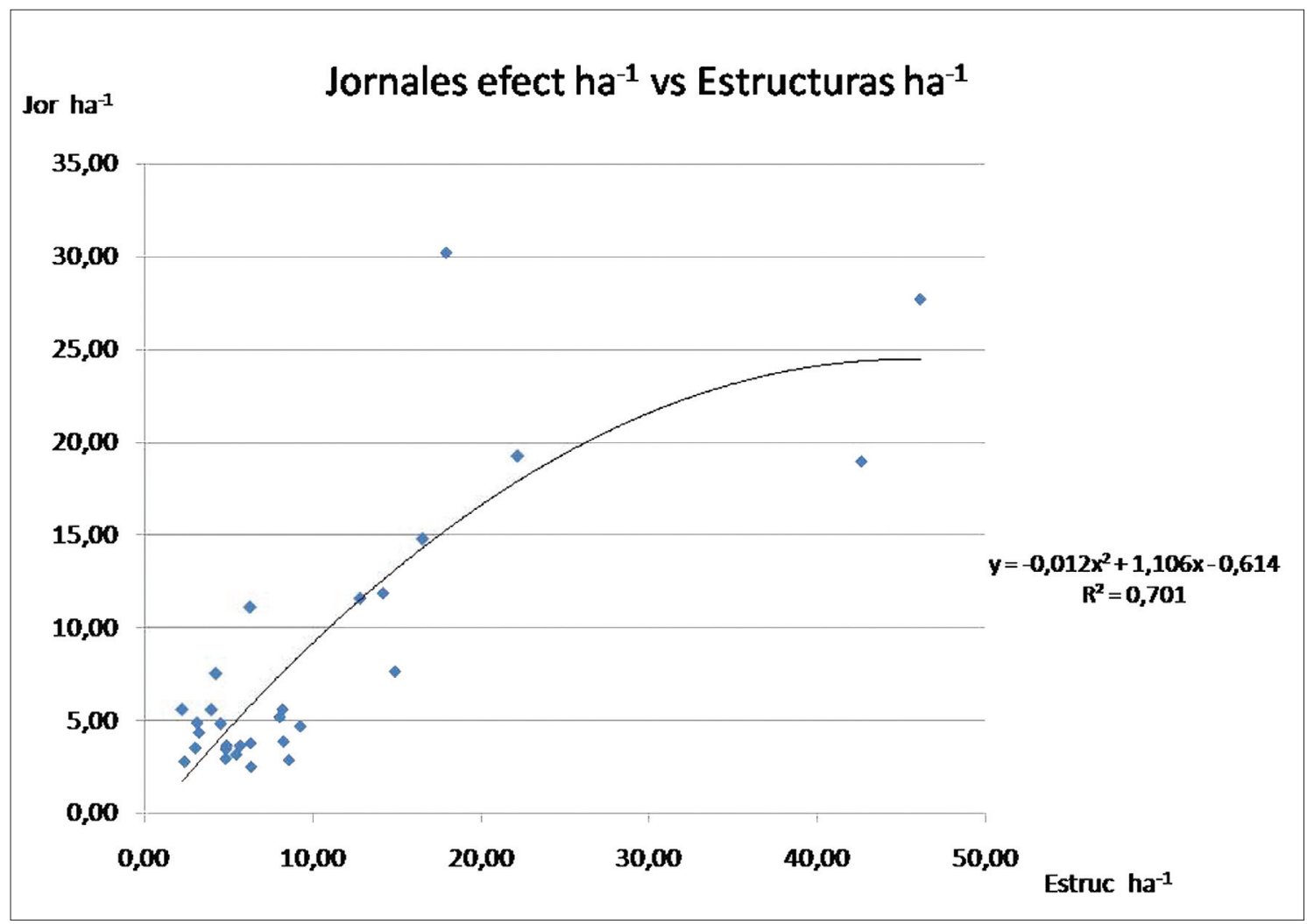

Figura 6. Representación gráfica de los rendimientos de las cuadrillas (jornadas efectivas ha ${ }^{-1}$ versus estructuras $h^{-1}$ ) y curva de regresión ajustada.

La curva de regresión ajustada entre los jornales efectivos y densidad de trabajo para cada medición se muestra en la Fig. 6, con expresión de la ecuación de la misma y su ajuste. La misma se ha obtenido para 458.34 ha y 3070 estructuras (albarradas y fajinas de $15 \mathrm{~m}$ ), en su mayoría a partir de bajas densidades de construcción. La tendencia inicial de la curva mantiene una pendiente elevada, pues los jornales efectivos necesarios son elevados. Por otro lado, a menor número de construcciones hidroló- 
gicas por hectárea mayor cantidad de hectáreas se ejecutan y a medida que aumenta el número de estructuras construidas por hectárea, las superficies ejecutadas son menores.

\section{Discusión}

En este apartado realizamos un ejercicio de discusión de los datos obtenidos en campo y lo relacionamos con el manual desarrollado.

La densidad de estructuras por hectárea, en el caso de fajinas, es menor a la aconsejable según la metodología (500 metros lineales ha-1 o 33.33 fajinas de 15 metros lineales $\left.\mathrm{ha}^{-1}\right)$. Este hecho se debe a la escasez de material para la construcción de las mismas. Sólo en algunos casos concretos se ha llegado a la densidad especificada en la metodología.

La densidad de albarradas ha dependido del número de cárcavas sobre el terreno, por lo que no se hace una valoración según datos. La necesidad de las mismas es variable según se presenta la topografía en cada zona.

La expresión de la Fig. 6 facilita la planificación de futuros trabajos similares, con posibilidad de plantear rendimientos ya en la fase de redacción del proyecto o plan. Los jornales expresados en este caso son de 7 horas de trabajo en campo, pudiéndose convertir a horas de trabajo por hectárea frente a densidad de trabajo en esa hectárea, según se demande en cada proyecto.

Para una mayor eficiencia del trabajo de cada cuadrilla, parece ideal valores intermedios o altos en la línea de tendencia expresada, donde un incremento en el número de estructuras requiere un incremento menor de jornales. Un valor de fajinas de 33 estructuras ha ${ }^{-1}$, según este manual, parece aconsejable a efectos de rendimientos de las cuadrillas.

Comparando los jornales efectivos ideales según la curva anterior, para las estructuras reales que se realizan en el monte, con los jornales efectivos reales por hectárea, nos marca la capacidad de mejora de cada cuadrilla en cada zona de trabajo.

Finalmente señalar que realizada la visita a las zonas trabajadas dos años después de su ejecución observamos las estructuras intactas, comprobándose la retención de sedimentos aguas arriba de las mismas así como el desarrollo de especies principalmente germinadoras, debido a la acumulación de semillas arrastradas por la escorrentía. Las estructuras en algunas zonas ya no son visibles por el desarrollo de vegetación. En casos muy puntuales de suelos poco consolidados se observa como el incorrecto sellado de la estructura al suelo ha dado lugar a regueras.

\section{Conclusiones}

- La redacción del manual de trabajo expuesto, empleado como base para la formación de los operarios, ha permitido una mayor eficacia de los recursos per- 
sonales y materiales disponibles así como la mayor funcionalidad de las estructuras realizadas.

- Las albarradas y fajinas construidas se configuran como estructuras eficaces frente al proceso de erosión hídrica. Siendo fundamental realizar un adecuado anclaje de las mismas para asegurar su permanencia, al menos hasta que la vegetación recolonice la zona, así como el correcto sellado de la estructura al suelo para evitar formación de regueras y garantizar su función de retención de sedimentos.

- Las dimensiones para la construcción de albarradas y fajinas, expuestas en este manual, se ajustan a las necesidades contra la erosión hídrica y mejor rendimiento del personal.

- Una localización adecuada de albarradas y fajinas repercute en el mejor control de la erosión hidrológica. Para su ejecución se seguirá "criterio de cuenca", localizando de arriba a abajo en topografía, los lugares más eficientes para su construcción.

- El número de estructuras por unidad de superficie está íntimamente relacionado con la cantidad de material disponible "in situ" para su construcción. El promedio de estructuras para el total de la superficie ejecutada es de 10.36 estructuras ha' $\mathrm{a}^{-1}$.

- Un incremento en la densidad de estructuras por hectárea, para valores de partida intermedios o altos, requiere un incremento menor de jornales de trabajo. Este hecho repercute en un mayor rendimiento de las cuadrillas de trabajo.

\section{Bibliografía}

Alloza, J.A. García-Barreda, S., Gimeno, T., Vallejo, R., Rojo, L., Martínez, A., 2013. Guía técnica para la gestión de montes quemados. Protocolos de actuación para la restauración de zonas quemadas con riesgo de desertificación. Madrid, España.

Bordón, P., Pérez-Laorga, E., Estruch, V.D., Rodrigo, E., 2012. Tabla de supervivencia de Pinus halepensis afectado por incendios forestales. Actas II Reunión sobre Sanidad Forestal. Cuad. Soc. Cienc. For. 36,161-165.

Chavarría, A., López de Diego, T., Vela, A., 2010. La restauración del área afectada por el incendio de El Rodenal de Guadalajara de julio de 2005. Foresta. 47-48 (Especial dedicado a Castilla la Mancha), 173-179.

Hernández, R., Romero, F., 2008. Manuales de desarrollo sostenible: 6. Criterios de restauración de zonas incendiadas. Fundación Banco Santander, Madrid. España. http://bib.cervantesvirtual.com/FichaObra.html?Ref=40744\&portal=113. Accesed. 05 January 2015.

J.A. Gómez, J.A., Taguas, E.V., Vanwalleghem, T., Giráldez, J.V., Sánchez, F., Ayuso, J.L., Lora, A., Mora, J., 2011. Criterios técnicos para el control de cárcavas, diseño de muros de retención y revegetación de paisajes agrarios. Manual del operador en inversiones no productivas. Sevilla, España.

Jordán, E., Valero, M.V., Rojo Núñez, I., González-Rincón, A., Espín-Navarro, F., Juanes, F.P., Masía, R., 2013. Empleo de albarradas en la restauración de áreas degradadas semiáridas en la región de Murcia. Actas V Congreso Forestal Español. 
Martínez-Menes, M., Rubio Granados, E., Oropeza Mota, J.L., Palacios Espinosa, C., 2009. Control de cárcavas. México.

Ministerio de Medio Ambiente, R. y M., 2008a. Inventario de tecnologías disponibles en España para lucha contra la desertificación. Utilización de fajinas para el control de la erosión en laderas y taludes. http:/www.magrama.gob.es/es/desarrollo-rural/temas/politicaforestal/desertificacion-restauracion-forestal/lucha-contra-la-desertificacion/lch_inv_ tec_obras_cauces.aspx. Accesed. 05 january 2015.

Ministerio de Medio Ambiente, R. y M., 2008b. Inventario Nacional de Tecnologías disponibles para la lucha contra la desertificación. Diseño de obras transversales: diques mixtos de mampostería y biomasa residual. http:/www.magrama.gob.es/ es/desarrollorural/temas/politica-forestal/desertificacion-restauracion-forestal/lucha-contra-la-desertificacion/lch_inv_tec_obras_cauces.aspx. Accesed. 05 january 2015.

Ramón Vallejo, V., Bautista Aguilar, S., Alloza Millán, J., 2009. Restauración de montes quemados en condiciones mediterráneas. Recursos Rurais do IBADER. Instituto de Biodiversidade Agraria e Desenvolvemento Rural.5, 55-60

Samuel-Francke, C., 2004. Manual de control de erosión. Chile. 